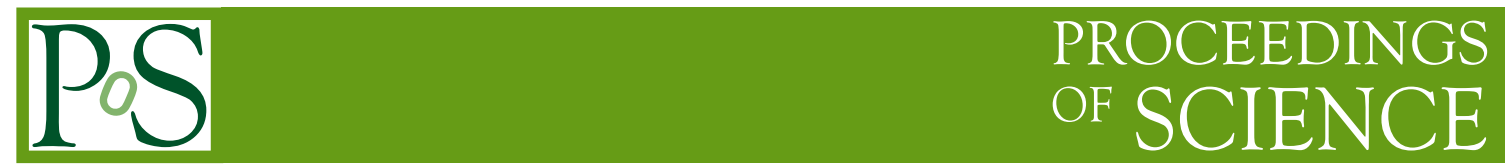

\title{
The First Large Application of MPPC: T2K Neutrino Beam Monitor INGRID
}

\section{Masashi Otani*}

Kyoto University, Japan

E-mail: masashi.o@scphys.kyoto-u.ac.jp

\begin{abstract}
We report design and performance of T2K neutrino beam monitor INGRID. INGRID has 9,592 channels which consists of scintillator, fiber, and MPPC. We assembled INGRID and tested all channels. RMS of MPPC gain is 5\% and light yield with cosmic-ray is larger than 19.4 photoelectrons.
\end{abstract}

International Workshop on New Photon Detectors PD09

June 24-26 2009

Shinshu University Matsumoto Japan

\footnotetext{
* Speaker.
} 


\section{Introduction}

The T2K(Tokai-to-Kamioka) experiment is long baseline neutrino oscillation experiment in Japan. J-PARC generates muon neutrino which is detected by near detector in J-PARC and SK(Super Kamiokande) located $295 \mathrm{~km}$ away from J-PARC(Fig 1 . The main goals of T2K are precise measurement of $v_{\mu}$ disappearance and the discovery of $v_{e}$ appearance.

One of the feature of T2K is the off axis neutrino beam. The neutrino beam direction is around 2.5 degrees away from SK direction. The off axis beam makes neutrino energy spectrum at SK sharply peaked at the oscillation maximum(Fig,2).

The peak of the energy spectrum, however, strongly depends on off-axis angle. Therefore the neutrino beam direction has to be monitored with high precision. INGRID(Interactive Neutrino GRID) monitors the neutrino beam direction with required precision day-by-day at the designed beam power.

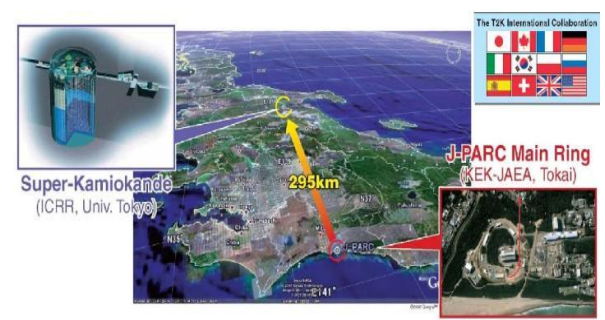

Figure 1: The T2K(Tokai-to-Kamioka) experiment is long base line neutrino oscillation experiment in Japan. Muon neutrino produced at J-PARC is observed at near detector at J-PARC and Super Kamiokande at $295 \mathrm{~km}$ away from JPARC.

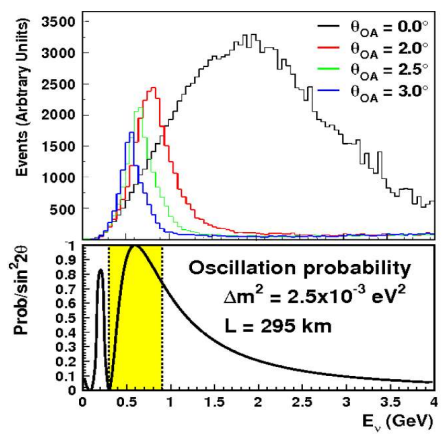

Figure 2: Top figure shows the beam neutrino energy spectrum expected at SK for several off axis angles. Bottom figure represents the oscillation probability at SK for oscillation parameters given in the figure. The neutrino beam is optimized to maximize the probability by choosing an appropriate off axis angle.

\section{Design of INGRID}

Because the beam neutrino is widely distributed at near detector hall, INGRID has to cover wide range. Hence INGRID consists of sixteen identical modules arranged in a cross around $\pm 5 \mathrm{~m}$ of the beam center(Fig 3 ).

INGRID module is a sandwich of nine irons and eleven scintillator tracking planes(Fig (4). Scintillator tracking plane consists of horizontal and vertical scintillator layers which are made of twenty-four scintillator bars. Scintillator bar is $120 \times 5 \times 1 \mathrm{~cm}^{3}$ and produced at FNAL[1]. Scintillation light is collected and transported with wave length shifting fiber which is inserted into a hole at the center of scintillator bar. One end of fiber is attached to MPPC(Multi Pixel Photon Counter). 
MPPC is the production of Hamamatsu Photonics K.K.. MPPC gain is $7.5 \times 10^{5}$ at $V_{\text {over }}=$ $1.5 V$ where $V_{\text {over }}$ is defined as $V-V_{b r}$. All performances except noise rate are characterized with $V_{\text {over }}$.

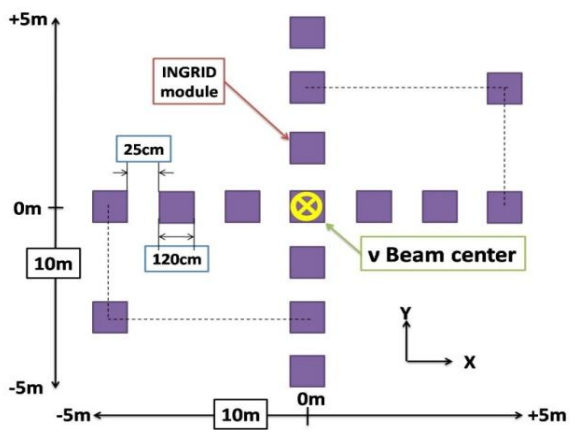

Figure 3: Schematic view of the INGRID detector configuration. Sixteen identical modules are placed around the beam center and cover \pm 5 $\mathrm{m}$ area. Seven modules are placed in horizontal direction, another seven are in vertical, and two are off axis position.

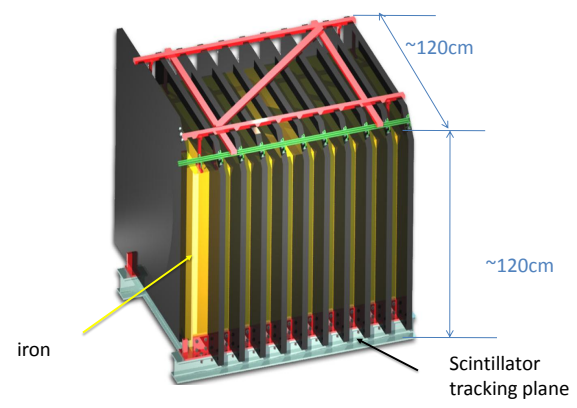

Figure 4: Schematic view of INGRID module. Module consists nine iron plates and eleven scintillator tracking planes. Iron plate at the most downstream position is missing.

\section{Performance of one channel}

In order to monitor the beam direction with required precision all channels must have high detection efficiency. In other words all channel should have large light yield. We measured the light yield of prototype of the channel with $3 \mathrm{GeV}$ electron beam at KEK FUJI test beam line. Fig 5 shows the setup of the measurement. Temperature is monitored with a temperature sensor and over voltage $\left(V_{\text {over }}\right)$ is adjusted to $1.4 \mathrm{~V}$.

Fig $[$ shows the distribution of the number of photoelectrons obtained by $3 \mathrm{GeV}$ electron beam. The light yield defined as the peak of distribution is 15.0 photoelectrons. The efficiency expected from Poisson distribution is $99.9 \%$ with 2.5 photoelectrons threshold. Consequently INGRID performance has met requirement.

\section{Assembly and Performance of INGRID}

We assembled all scintillator planes from August 2008 to December 2008. Before installtion of all MPPCs, we measured the basic performances of all MPPCs [2]. Using this data, $V_{\text {over }}$ is set to $1.5 \mathrm{~V}$. Just after assemly we checked the performances of all 9,592 channels. First we checked MPPC gain and then we measured light yield with cosmic-ray.

First of all we checked ADC distribution of all channels by eye(Fig 7). After that we calculated the distance between pedestal peak and 1 p.e. peak to obtain MPPC gain by the unit of ADC counts. RMS of MPPC gain is $5 \%$ (Fig 8 ).

Finally we measured light yield of all channels with cosmic-ray. We piled up four scintillator planes and used top and bottom one as the trigger counter. In this analysis we defined light yield 


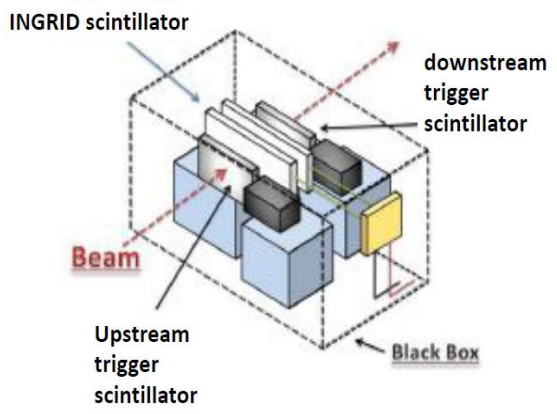

Figure 5: Experimental setup of the measurement of light yield with $3 \mathrm{GeV}$ electron beam at KEK FUJI test beam line. Electron beam events are triggered with upstream and downstream scintillation counter.

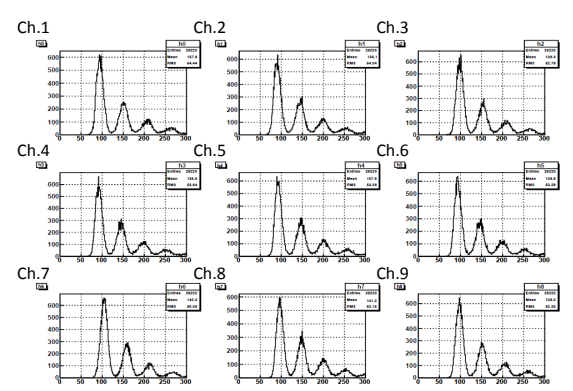

Figure 7: Examples of ADC distribution of MPPC darknoise. The data was taken with periodic trigger. First large peak is pedestal and second one is 1 p.e. peak and so on. All distributions are almost identical.

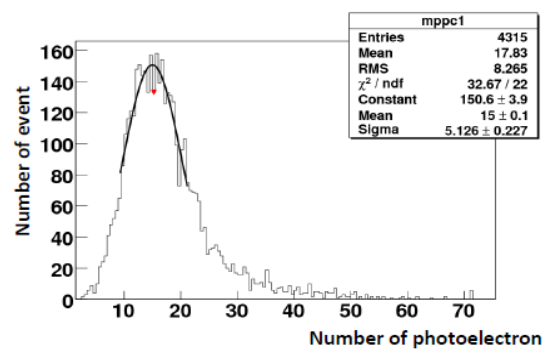

Figure 6: Light yield distribution with $3 \mathrm{GeV}$ electron beam. Light yield defined as the peak of distribution is 15.0 photoelectrons.

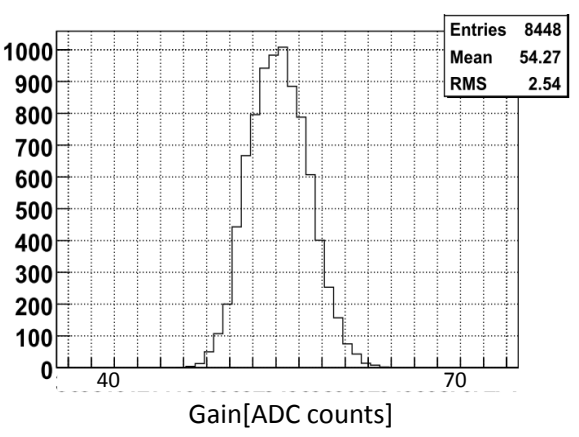

Figure 8: The distribution of MPPC gain with the unit of ADC counts. RMS of gain is $5 \%$.

as the mean of the number of photoelectrons because the number of events is not enough to fit the peak of the distribution. Light yield of all channels is larger than 19.4 photoelectrons.

From June 2009 to October we assembled and installed all INGRID modules. T2K neutrino beam commissioning will start from October 2009.

\section{Summary}

We designed INGRID for the T2K neutrino beam monitor and tested prototype channel of it with the $3 \mathrm{GeV}$ electron beam. One channel consists of scintillator bar, fiber and MPPC. The light yield of prototype channel is 15.0 photoelectrons which is high enough.

We assembled INGRID and tested all channels. RMS of gain is 5\% and light yield of all channels is larger than 19.4 photoelectrons. Consequently we expect INGRID has required peformance and observe T2K first neutrino event at beam commissioning which will start from October 2009. 


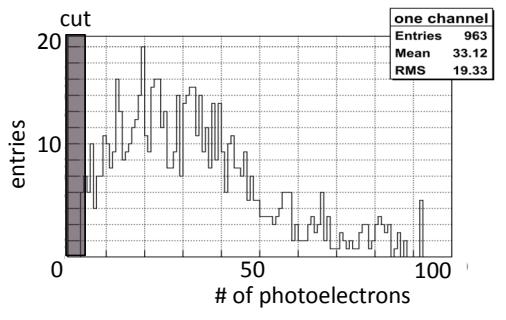

Figure 9: The distribution of light yield with cosmic-ray. We cut the events which have smaller than 10 photoelectrons in order to remove the MPPC noise event amplified by cross talk and after pulse. Light yield is defined as the mean of the distribution.

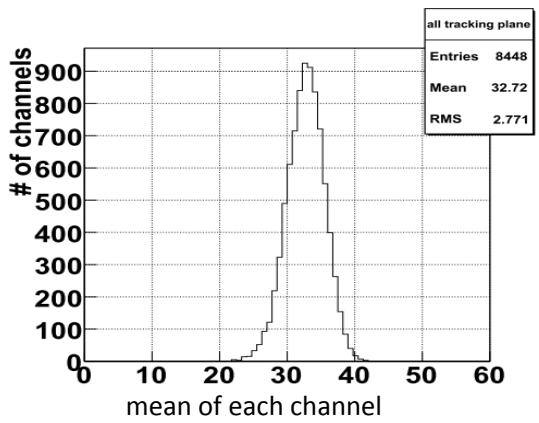

Figure 10: Light yield of each channel. Light yield is larger than 19.4 photoelectrons.

\section{Acknowledgments}

It is a pleasure to thank KEK FUJI test beam line group. We are grateful to Mechanical Support Co. for helping assembly of INGRID.

\section{References}

[1] A.Pla-Dalmau,A.D.Bross,and V.V.Rykalin, FERMILAB-CONF-03-3180E.

[2] M.Yokoyama et al."Mass production test of Hamamatsu MPPC for T2K neutrino oscillation experiment", arXiv:0807.3147 